\title{
Using Bar Infrared Spectra and Coincidence Indexes to Study the Diversity of Solid Cyanuric Acid Structures
}

\author{
Marcela C. B. G. Nilo, ${ }^{a}$ Thais G. Simões ${ }^{a}$ and Claudio Costa Neto ${ }^{*, b}$ \\ ${ }^{a}$ Fundação Ataulpho de Paiva (FAP), Av. Pedro II, 260, São Cristóvão, 20941-000 Rio de Janeiro-RJ, Brazil \\ ${ }^{b}$ Polo de Xistoquímica, Instituto de Química, Universidade Federal do Rio de Janeiro (UFRJ), \\ Rua Hélio de Almeida, 40, Cidade Universitária, 21941-614 Rio de Janeiro-RJ, Brazil
}

\begin{abstract}
A general method was developed for studying the diversity of individuals in a population based on the diversity of infrared spectra of solid cyanuric acid analytes obtained from various reactions of trichlorocyanuric acid. This method first generates infrared bar spectra for the analytes and then measures the coincidence and continence among pairs of the spectral peaks via confrontation matrices. Class markers are established to characterize analyte classes. Possible correlations among the employed reaction conditions and the nature of the produced solids, which are based on their infrared bar spectra, are discussed. The method of coincidence may be useful for characterizing polymorphs, particularly those of active pharmaceutical ingredients (APIs). The method may also be extended to define the homogeneity of solid analytes. The ANALIN module of the ANALOR software suite running on a dBase platform is used to generate the bar infrared spectra and to handle all calculations.
\end{abstract}

Keywords: infrared bar spectra, coincidence indexes, cyanuric acid, trichloroisocyanuric acid, polymorphism

\section{Introduction}

This study began with the authors' observation of ca. 30 infrared printed (common) spectra generated directly from a spectrometer of the white precipitate resulting from a group of reactions with trichloroisocyanuric acid. Although all these products are believed to be cyanuric acid, a diversity of infrared spectra was observed. Notably, some of these spectra were similar, but not identical, whereas others were more diverse. Initially, simply defining the existing differences (were all of the products the same?) was deemed necessary. Later, the quantification of these differences was preferred in order to determine a rationale that could correlate them. The objective of this paper is to present results on the study of the diversity of infrared spectra of cyanuric acid obtained from various reactions using trichloroisocyanuric acid while adhering to two main directions: (i) generating the bar spectra of the various products and mapping the frequencies in order to group the analytes into classes, as well as concurrently determining the correlations between the reaction conditions and the structure (the class) of the solids produced. Classes were

*e-mail: ccostaneto@terra.com.br characterized by groups of frequencies, the 5-frequency markers; and (ii) measuring the differences among the spectra based on the coincidence and continence of frequencies among the analytes, as calculated from their infrared bar spectra, and exploring the results obtained. The method developed aims to represent the differences among the spectra by numbers (indexes), with these differences being large or (very) small.

Cyanuric acid has been known since a paper from the nineteenth century (1829) by Friedrich Wöhler. ${ }^{1}$ Wöhler produced cyanuric acid by heating urea or uric acid. The so-called cyanuric acid exists in a tautomeric equilibrium with isocyanuric acid. The three "phenolic" hydroxyl groups in cyanuric acid are responsible for its (weakly) acidic character (the $\mathrm{pH}$ of a saturated solution is $4.5-5.0) ;{ }^{2}$ consequently, it is the preferred tautomer in basic solution. The literature indicates that isocyanuric acid is the predominant isomer in the solid phase but is also present in solution. ${ }^{3}$

In the current literature, both cyanuric and isocyanuric acids are referred to as cyanuric acid. Chemical Abstracts/ SciFinder define one Chemical Abstracts Service (CAS) registry number (08-80-5) for the isomers cyanuric acid (2,4,6-trihydroxy-1,3-5-triazine) and isocyanuric acid 
(1,3,5-triazine-2,4,6-trione). However, another CAS registry number, 135581-61-2, is given to the former species under the name of 1,3,5-triazine-2,4,6-triol, despite isocyanuric acid also being given as a synonym for this molecule. Cyanuric/isocyanuric acid will be represented in this paper by the three letter abbreviation ACN and trichloroisocyanuric acid by TCI (other abbreviations for trichloroisocyanuric acid can be found in the literature, such as $\mathrm{TCCA}^{4}$ and $\mathrm{TICA}^{5}$ ).

A very complete account on cyanuric acid is presented by Wojtowicz. ${ }^{2,6}$

From cyanuric acid, trichloroisocyanuric acid (CAS 87-90-1) can be prepared by direct chlorination with chlorine gas. ${ }^{7}$ This compound is extensively used as a disinfectant agent for swimming pools (algicide and bactericide). ${ }^{8}$ Presently, TCI is also widely employed as an effective chlorinating reagent for various chemical reactions, such as the $\alpha$-chlorination of carbonyl derivatives, ${ }^{9}$ the production of nitriles from amines, ${ }^{10}$ the halogenation and epoxidation of alkenes, ${ }^{11}$ and the chlorination of aromatic systems, ${ }^{5,12}$ among others; extensive reviews on the reactions of TCI have been offered by Tilstan and Weinmann, ${ }^{4}$ Cunha and Ferreira ${ }^{13}$ and Mendonça and Mattos. ${ }^{14}$ Figure 1 shows the chemical structures of these molecules.<smiles>O=c1n(Cl)c(=O)n(Cl)c(=O)n1Cl</smiles><smiles>O=c1[nH]c(=O)[nH]c(=O)[nH]1</smiles><smiles>Oc1nc(O)nc(O)n1</smiles>

$N, N^{\prime}, N^{\prime \prime}$-trichloroisocyanuric acid

$$
\text { Isocyanuric acid }
$$

Figure 1. Chemical structures of trichloroisocyanuric acid, isocyanuric acid and cyanuric acid.

Contributions to the assignment of infrared absorption frequencies to chemical moieties of cyanuric acid can be found in the literature. These assignments, particularly those related to hydrogen bonds, are important for establishing the structures of the solid, as well as for build correlation maps of frequencies among analytes. In this context, Newman and Badger ${ }^{15}$ presented in 1952 a very complete analysis of a set of 16 peak frequencies for a sample of sublimed cyanuric acid and then assigned them to their corresponding chemical moieties.

Other, albeit less informative, assignments can be found in the literature, such as Padgett and Hamner, ${ }^{16}$ Loughran et al. ${ }^{17}$ Chen et al.,${ }^{18}$ and Surinwong et al. ${ }^{19}$ References can also be found in the literature regarding polymorphs of cyanuric acid derivatives, such as Barnett et al. ${ }^{20}$ who discuss the polymorphism in hydrogen bonded adducts of cyanuric acid-bis(4-pyridyl)ethane, and

Yang et al.,${ }^{21}$ who address the effects of cyanuric acid on the polymorphism of poly(butylene adipate).

\section{Experimental}

This paper addresses a study on the white precipitate produced in the halogenation of the double bond of methyl acrylate with TCI and a halogen salt, following a similar rationale used by Tozetti et al. ${ }^{22}$ for the bromination of alkenes.

With the stoichiometric coefficients added, the complete equation for the bromination of methyl acrylate with TCI and potassium bromide may be written as

$3 \mathrm{CH}_{2}=\mathrm{CHC}(\mathrm{O}) \mathrm{OCH}_{3}+2$ trichloroisocyanuric acid + $6 \mathrm{KBr} \rightarrow 3 \mathrm{CH}_{2} \mathrm{Br}-\mathrm{CHBrC}(=\mathrm{O}) \mathrm{OCH}_{3}+6 \mathrm{KCl}+$ 2 isocyanuric acid

A group of 35 reactions of trichloroisocyanuric acid with methyl acrylate or acrylonitrile and a bromide or chloride salt of potassium or ammonium using acetone and water or ammonia as solvents at different temperatures were selected to study the effect of these reagents and conditions on the yield and purity of the methyl 2,3-dibromopropionate product. The reaction conditions used are shown in Table 1.

As a complementary study, the infrared spectra of the white precipitates produced in all the reactions were recorded and became the focus of the present work. The reactions conducted at different conditions produced, in all cases, a white precipitate.

In a general procedure, a solution of $0.050 \mathrm{~mol}$ of the acrylate derivative dissolved in $25 \mathrm{~mL}$ of acetone was added to an initial solution of $0.116 \mathrm{~mol}$ of the salt dissolved in $20 \mathrm{~mL}$ of water. The two solutions were mixed to generate a final homogeneous solution and subjected to a defined temperature. Next, a solution of $0.017 \mathrm{~mol}$ of TCI dissolved in $25 \mathrm{~mL}$ of acetone was added dropwise with agitation to the previous solution over $1 \mathrm{~h}$. The mixture was continually agitated for an additional hour and then filtered. The precipitate can be easily solubilized in hot water and reprecipitated by cooling (the solubility of cyanuric acid in water is $0.2 \%$ at $25^{\circ} \mathrm{C}$ and $2.6 \%$ at $90{ }^{\circ} \mathrm{C}$ ). ${ }^{4}$ The filtrate was concentrated and left to cool, leading to the production of a second crop.

The above procedure differs for the one proposed by Tozetti et al.,$^{22}$ mainly in the way TCI is introduced: in the Tozetti et al..$^{22}$ procedure, TCI is added as a powdered solid directly into the reaction media, whereas in most of the reaction discussed in this text, TCI was added in a solution of acetone. Reactions 17.00 to $24.00,26.00$ to 
Table 1. Trichloroisocyanuric acid reaction conditions

\begin{tabular}{|c|c|c|c|c|c|c|c|}
\hline \multirow{2}{*}{ Reaction } & \multicolumn{2}{|c|}{ Halogen acceptor } & \multirow{2}{*}{$\mathrm{TCI} / \mathrm{mol}$} & \multicolumn{2}{|c|}{ Halogen salt } & \multirow{2}{*}{ Temperature $/{ }^{\circ} \mathrm{C}$} & \multirow{2}{*}{ Medium/solvent } \\
\hline & Acceptor & No. of moles & & Salt & No. of moles & & \\
\hline 2.00 & AcrMet & 0.056 & 0.020 & $\mathrm{KBr}$ & 0.116 & r.t./ca. $28+$ & $\mathrm{H}_{2} \mathrm{O}$ /acetone \\
\hline $3.00 / 4.00 / 205.00$ & AcrMet & 0.050 & 0.017 & $\mathrm{KBr}$ & 0.100 & r.t./ca. $28+$ & $\mathrm{H}_{2} \mathrm{O} /$ acetone \\
\hline 26.00 & AcrMet & 0.050 & 0.017 & $\mathrm{KBr}$ & 0.116 & 25 & $\mathrm{H}_{2} \mathrm{O} /$ acetone \\
\hline 20.00 & AcrMet & 0.050 & 0.017 & $\mathrm{KBr}$ & 0.116 & 0 & $\mathrm{H}_{2} \mathrm{O} /$ acetone \\
\hline 21.00 & AcrMet & 0.050 & 0.017 & $\mathrm{KBr}$ & 0.116 & 0 & $\mathrm{NH}_{4} \mathrm{OH}_{\mathrm{aq}} /$ acetone \\
\hline 10.00 & AcrMet & 0.050 & 0.017 & $\mathrm{KBr}$ & 0.116 & r.t./ca. $28+$ & $\mathrm{NH}_{4} \mathrm{OH} /$ acetone \\
\hline 11.00 & - & 0.000 & 0.017 & $\mathrm{KBr}$ & 0.116 & r.t./ca. $28+$ & $\mathrm{NH}_{4} \mathrm{OH} /$ acetone \\
\hline $5.00 / 6.00 / 7.00 / 8.00$ & AcrMet & 0.050 & 0.017 & $\mathrm{KCl}$ & 0.100 & r.t./ca. $28+$ & $\mathrm{NH}_{4} \mathrm{OH} /$ acetone \\
\hline 9.00 & - & 0.000 & 0.017 & $\mathrm{KCl}$ & 0.100 & r.t./ca. $28+$ & $\mathrm{NH}_{4} \mathrm{OH} /$ acetone \\
\hline 27.00 & AcrMet & 0.050 & 0.017 & $\mathrm{KCl}$ & 0.100 & 25 & $\mathrm{H}_{2} \mathrm{O} /$ acetone \\
\hline 214.00 & AcrMet & 0.050 & 0.017 & $\mathrm{KCl}$ & 0.100 & r.t./ca. $28+$ & $\mathrm{H}_{2} \mathrm{O} /$ acetone \\
\hline $212.00 / 215.00$ & AcrNit & 0.050 & 0.017 & $\mathrm{KCl}$ & 0.100 & r.t./ca. $28+$ & $\mathrm{H}_{2} \mathrm{O}$ /acetone \\
\hline 14.00 & AcrMet & 0.050 & 0.017 & $\mathrm{NH}_{4} \mathrm{Br}$ & 0.100 & r.t./ca. $28+$ & $\mathrm{H}_{2} \mathrm{O}$ /acetone \\
\hline 28.00 & AcrMet & 0.111 & 0.371 & $\mathrm{NH}_{4} \mathrm{Br}$ & 0.222 & 25 & $\mathrm{H}_{2} \mathrm{O} /$ acetone \\
\hline $17.00 / 22.00$ & AcrMet & 0.050 & 0.017 & $\mathrm{NH}_{4} \mathrm{Br}$ & 0.100 & 0 & $\mathrm{H}_{2} \mathrm{O} /$ acetone \\
\hline 33.00 & AcrMet & 1.110 & 0.372 & $\mathrm{NH}_{4} \mathrm{Br}$ & 2.222 & 25 & $\mathrm{H}_{2} \mathrm{O} /$ acetone \\
\hline 15.00 & AcrMet & 0.050 & 0.017 & $\mathrm{NH}_{4} \mathrm{Br}$ & 0.100 & r.t./ca. $28+$ & $\mathrm{NH}_{4} \mathrm{OH}_{\mathrm{aq}}$ /acetone \\
\hline $23.00 / 18.00$ & AcrMet & 0.050 & 0.017 & $\mathrm{NH}_{4} \mathrm{Br}$ & 0.100 & 0 & $\mathrm{NH}_{4} \mathrm{OH}_{\mathrm{aq}} /$ acetone \\
\hline 19.00 & AcrMet & 0.050 & 0.017 & $\mathrm{NH}_{4} \mathrm{Br}$ & 0.100 & 0 & $\mathrm{NH}_{4} \mathrm{OH} /$ acetone \\
\hline 16.00 & AcrMet & 0.050 & 0.017 & $\mathrm{NH}_{4} \mathrm{Br}$ & 0.100 & r.t./ca. $28+$ & $\mathrm{NH}_{4} \mathrm{OH} /$ acetone \\
\hline 12.00 & - & 0.000 & 0.017 & - & 0.000 & r.t./ca. $28+$ & $\mathrm{NH}_{4} \mathrm{OH} /$ acetone \\
\hline 47.00 & - & 0.000 & 0.372 & - & 0.000 & 25 & $\mathrm{NH}_{4} \mathrm{OH} /$ acetone \\
\hline 24.00 & - & 0.000 & 0.017 & - & 0.000 & -10 & $\mathrm{NH}_{4} \mathrm{OH}_{\mathrm{aq}} /$ acetone \\
\hline 25.00 & - & 0.000 & 0.017 & - & 0.000 & r.t./ca. $28+$ & $\mathrm{NH}_{4} \mathrm{OH}_{\mathrm{aq}}$ /acetone \\
\hline 31.00 & AcrMet & 0.000 & 0.017 & - & 0.000 & -15 & $\mathrm{NH}_{4} \mathrm{OH}$ \\
\hline 210.00 & AcrMet & 0.050 & 0.017 & - & 0.000 & r.t./ca. $28+$ & $\mathrm{HCl} / \mathrm{H}_{2} \mathrm{O} /$ acetone \\
\hline 29.00 & AcrMet & 0.111 & 0.371 & $\mathrm{NH}_{4} \mathrm{Cl}$ & 0.222 & 25 & $\mathrm{H}_{2} \mathrm{O}$ /acetone \\
\hline
\end{tabular}

TCI: trichloroisocyanuric acid; AcrMet: methyl acrylate; AcrNit: acrylonitrile; r.t.: room temperature.

29.00 and 31.00 were conducted in $100 \mathrm{~mL}$ Easy-Max Mettler-Toledo equipment, and reactions 33.00 and 47.00 were part of a study on reaction calorimetry that used the Mettler-Toledo RC1 equipment. Both Easy-Max and RC1 provide very strict control of temperature (the reaction is quite exothermic), which differs from reactions 3.00 and 205.00, for which the TCI was added as a lump (as recommend in the procedure of Tozetti et al. $)^{22}$ to a normal reaction flask at room temperature.

\section{Equipment and instruments}

All infrared analysis was performed using standard $\mathrm{KBr}$ disks. The instrument used was a Nicolet 6700 -Fourier transform infrared (FTIR) spectrometer operating under default conditions. The infrared region used in this paper extends from 3600 to $2500 \mathrm{~cm}^{-1}$ (region A'), a subregion within the A region $\left(4000-2500 \mathrm{~cm}^{-1}\right)$. This region is chosen because the peaks in the bar spectra in the region of $4000-3600 \mathrm{~cm}^{-1}$ are always very noisy and, for the purposes of this paper, do not add any significant information (see Figure 2).

The false intensity plots (FI-plots or $\phi$-plots) were drawn with Microcal-Origin $6.0^{23}$ software from data generated by ANALIN.

The ${ }^{13} \mathrm{C}$ nuclear magnetic resonance (NMR) spectra of the samples were obtained on a Bruker 300 NMR spectrometer using dimethylsulfoxide- $d_{6}$ (DMSO) as the solvent.

\section{Chemicals}

Methyl acrylate, cyanuric acid, and dimethylsulfoxide- $d_{6}$ were acquired from Sigma-Aldrich; potassium chloride, 


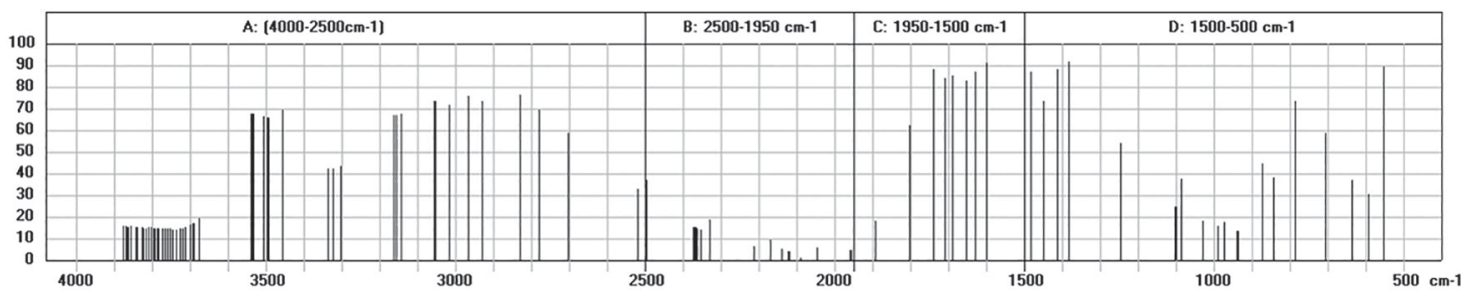

Figure 2. Infrared bar spectra of cyanuric acid analyte 4.04, representing a typical infrared bar spectra produced by ANALIN using the three consecutive points algorithm. Note the noise above $3600 \mathrm{~cm}^{-1}$; for the discussions in this paper, this region is discarded.

potassium bromide, ammonium chloride, ammonium bromide and acetone of pure grades, from Vetec Química Fina; and ammonia, aqueous solution containing $24 \% \mathrm{NH}_{3}$, from Isofar Indústria e Comércio de Produtos Químicos. Trichloroisocyanuric acid was supplied by HTH (a swimming pool supplier) and was said to be $>99 \%$ pure.

\section{Methodology}

\section{The infrared bar spectra}

The infrared bar spectrum is a graphic representation of a subset of the array of frequency-intensity values generated by an infrared spectrometer. It contains only bars corresponding to the peaks of the spectrum. Similar to the conventional way of representing an infrared spectrum, wavenumbers are shown on the abscissa and intensities on the ordinate (as transmittances); however, different from most conventional representation, intensities are normalized to $100 \%$ (the highest value of intensity), and the peaks are shown extending upwards. The bar spectrum is a discrete form of spectral representation rather than the continuous conventional display of spectra, in which all of the points next to each other are linked.

The output of an infrared instrument is a table that contains ca. 4500 points provided in ".csv" format (Excel). The intensity values can be set as a simple one-dimensional array of data from which the subset of peaks can be extracted by software using proper algorithms.

Simple algorithms can be used to extract peaks from the one-dimensional array of intensity values generated by an infrared spectrometer, such as the three consecutive points algorithm, in which the intensity of the middle point of three consecutive points of a linear array is compared with that of the other two points: if it is greater than the other two, then this constitutes a peak (maximum) that is registered for the bar spectrum as an intensity with the corresponding frequency. The three consecutive points algorithm is simple to implement, can almost instantly screen the whole set of peaks generated by an infrared spectrometer and extract the subset of peaks and is robust in use; thus, it was chosen to generate the bar spectra in this paper.
A great advantage of using bar spectra is the ease with which they work with software. The bar infrared spectra were generated directly from the output of an FTIR spectrometer. This process can be achieved quickly using version 2 of the ANALIN module of the ANALOR ${ }^{24}$ software suite running on a dBase platform.

Most of the commercial FTIR instruments designed to produce infrared spectra for routine operations may show a variation up to $\pm 2 \mathrm{~cm}^{-1}$ in a frequency value. A comparison between spectra must always account for this "error". A graphic example of a bar infrared spectrum generated by ANALIN can be seen in Figure 2.

\section{False intensity plots}

A false intensity plot is defined as a frequency-intensity (transmittance) plot with a proper format in which the different peak intensities of the spectrum provided by the spectrometer are substituted by a constant intensity value. False intensities can be assigned to all the analytes of the same class or to each analyte of a given class. In the first case, the various classes can be represented simultaneously in the same graph, whereas in the second, observing and comparing the distribution of the analytes of each class is possible. Examples can be seen later in the Results and Discussion section under the heading "Maps of analyte frequency distribution".

The coincidence and continence indexes between two infrared bar spectra

The comparison between two infrared bar spectra may be accomplished numerically as graphs or indexes and displayed in two ways:

First, two bar spectra may be initially displayed graphically. This procedure uses both absorption frequencies and intensities to display the two spectra on a computer. In the graphic display mode, many operations can be further performed on the two spectra, for instance, eliminating all of the peaks of the analyte that are not consistent with the reference (intensities are not considered). This process leaves a residue spectrum (as a 
new analyte spectrum) with only the peaks in consonance with the reference.

A second mode is to calculate the coincidence between the peaks of the spectrum of an analyte and those of a reference. The greater the coincidence, the greater the similarity between the two. As a particular case of coincidence, the continence of peaks in the reference spectrum within the analyte spectrum can also be calculated. These comparisons are based on frequency values only (intensities have not been considered in the present version of the software).

The analysis of the coincidence of peaks of two spectra can be performed in many ways, and depending on how they are considered, different indexes can be established. The following indexes are defined in this paper:

The single coincidence index (SCI) relates the ratio of the number of coincident peaks between the analyte and the reference to the number of peaks of the reference. An index relating the same number of coincident peaks between the analyte and the reference to the number of peaks of the analyte could also be practical. Here, a group of indexes relating these types of coincidence can now be expressed based on the following parameters: the total number of peaks of the analyte (a); the total number of peaks of the reference (b); the number of peaks of the analyte coinciding with those of the reference (c); the number of peaks of the reference coinciding with those of the analyte (d); and the error interval for the peak values allowed for the calculations (this error interval is added to the calculations to handle the imprecision of the spectrometer when assigning frequency values to the peaks).

A single coincidence index relative to the analyte $(\%)$ : SCIana $=(\mathrm{c} / \mathrm{a}) \times 100$

A single coincidence index relative to the reference $(\%)$ : SCIref $=(\mathrm{d} / \mathrm{b}) \times 100$

These two indexes indicate (i) the number of peaks of the analyte coincident with peaks of the reference relative to the number of peaks of the analyte; and (ii) the number of peaks of the reference coincident with peaks of the analyte relative to the number of peaks of the reference. The index related to the reference seems to be more appropriate for the analysis of the results: if the index has a value of 1 (or $100 \%$ ), then all of the peaks of the reference are contained in the spectrum of the analyte; by contrast, if the index is 0 (or 0\%), even at a large error interval, no coincidence exists between the analyte and reference, and they are probably different species. For any value between $0(0 \%)$ and 1 $(100 \%)$, some resemblance may exist between the analyte and reference, and the higher the indexes, the more similar the analytes should be. A similar analysis can be made for the single coincidence index related to the analyte. Indexes should always indicate the error used to calculate them.

A double coincidence index (DCI) can be determined by taking into account the coincident plus the non-coincident (NDCI) peaks of both the analyte and the reference, referred to the total number of peaks (peaks of analyte plus peaks of reference). The DCI and NDCI can be calculated using the following relations:

$\mathrm{DCI}=[(\mathrm{c}+\mathrm{d}) /(\mathrm{a}+\mathrm{b})] \times 100$

$\mathrm{NDCI}=100-\mathrm{DCI}$

The DCI index gives a better idea (when compared with the SCI) of how similar two spectra are.

To facilitate the understanding of the coincidence/ continence concept, a pictorial representation of the indexes is shown in Figure 3.

\section{Results and Discussion}

From an initial observation of a set of 30 (printed) infrared spectra of the white precipitates (later extended to 151 ), grouping the spectra visually (empirically and roughly) into four classes (called VA-classes)

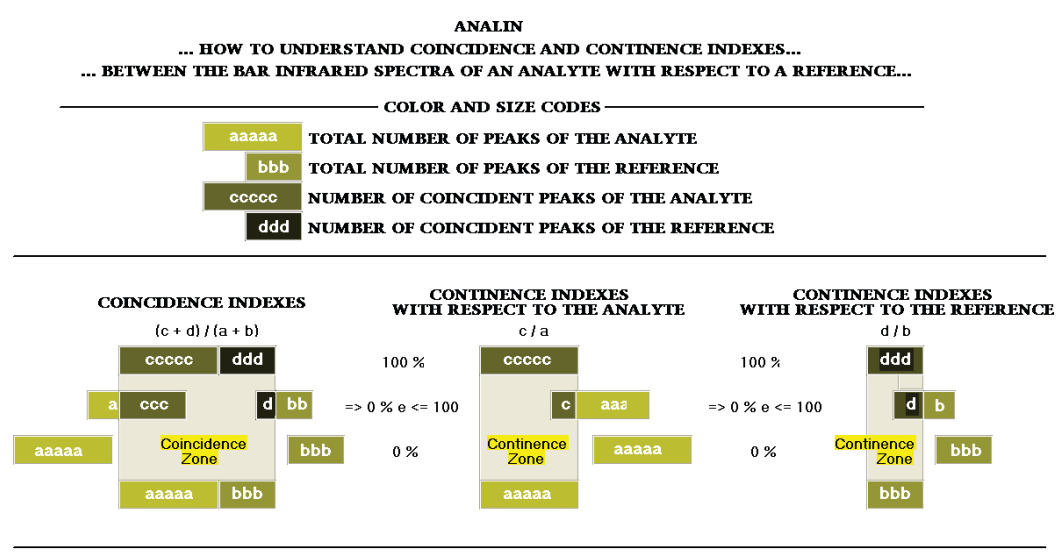

Figure 3. Pictorial representation of the indexes of the coincidence and continence between two infrared bar spectra. 


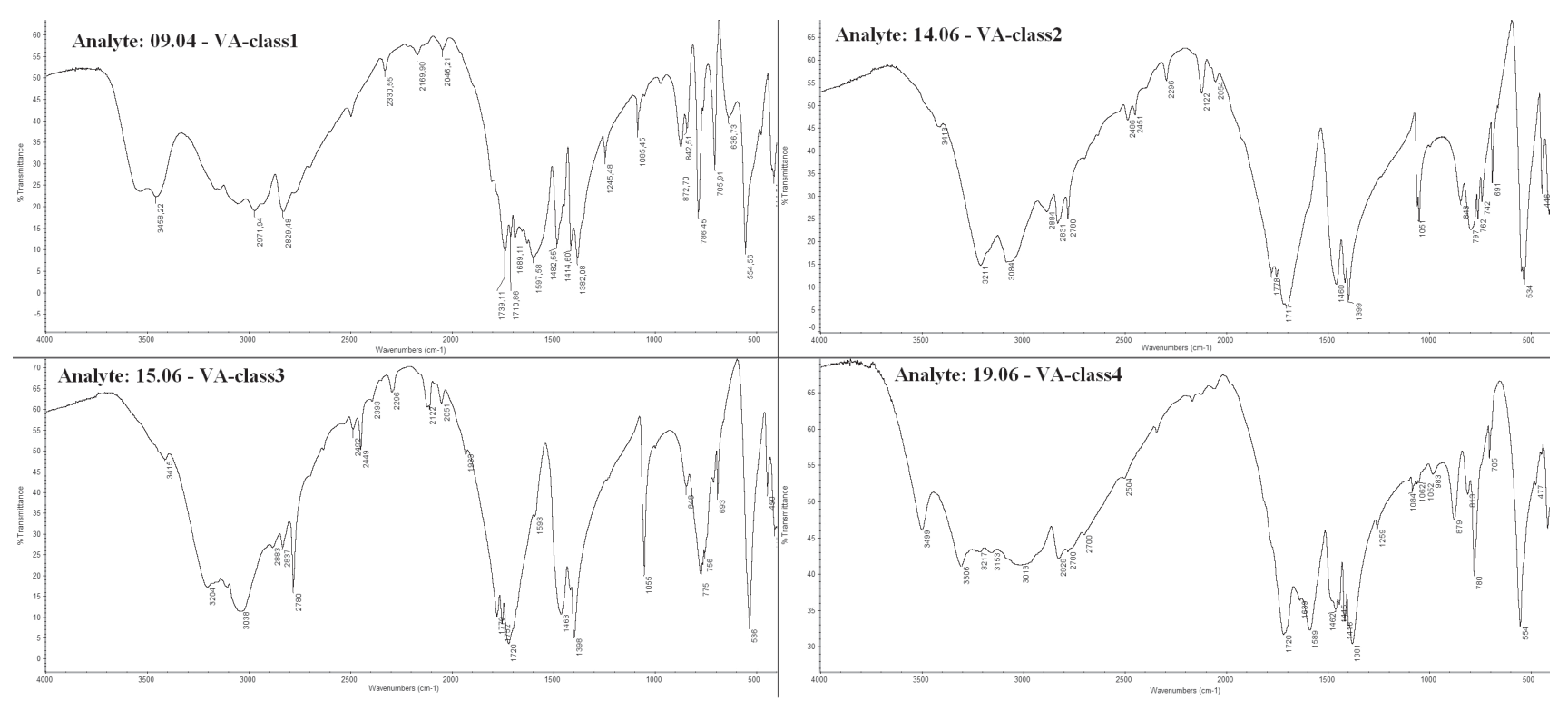

Figure 4. Infrared spectra of the cyanuric acid VA-classes derived from trichloroisocyanuric acid reactions.

based on the visual pattern presented by the peaks in region $3600-2500 \mathrm{~cm}^{-1}$ was possible. Figure 4 shows a representative infrared spectrum of each class.

These spectra are clearly different, and the spectra within each class are similar but not equal. The comparison between two spectra could be established by examining the coincidence between the peaks of both spectra. However, obtaining (good) quantitative data by comparing two printed spectra (as the ones above) using either method is difficult if not impossible. Conversely, a quantitative measurement of the coincidence or continence between two spectra may be easily and quickly accomplished using bar spectra and appropriate algorithms. In both cases, a relation between two quantities of the same species is measured, with the ratio between the two being represented by a number (indexes).

Other methods for comparatively quantifying infrared spectra are described in the literature. For instance, Luo et al. ${ }^{25}$ used three-dimensional Hirshfeld surfaces and two-dimensional fingerprint plots to quantitatively compare the polymorphs of pyrazinamide.

\section{${ }^{13} \mathrm{C}$ NMR of the white precipitates}

Although all these precipitates are expected to be cyanuric acid, because the infrared spectra are quite dissimilar, the first question to be answered was whether or not they are all really the same chemical species. A prompt answer was provided by obtaining ${ }^{13} \mathrm{C}$ NMR spectra of the analytes dissolved in DMSO. The chemical shifts recorded for these analytes are presented in Table 2 .

Table 2 shows that the spectra of the analytes soluble in DMSO exhibited only one peak at $149.07 \pm 0.06 \mathrm{ppm}$,
Table 2. ${ }^{13} \mathrm{C}$ NMR results for the analytes produced in the reactions listed in Table 1

\begin{tabular}{lccc}
\hline Analyte & VA-class & $\begin{array}{c}{ }^{13} \text { C NMR } \\
\text { peaks / ppm }\end{array}$ & Solvent \\
\hline 04.10 & 1 & insoluble & - \\
205.10 & 1 & insoluble & - \\
09.04 & 1 & insoluble & - \\
14.06 & 2 & 149.01 & DMSO \\
17.05 & 2 & 149.11 & DMSO \\
17.06 & 2,3 & 149.07 & DMSO \\
33.09 & 2 & 149.13 & DMSO \\
15.06 & 3 & 149.04 & DMSO \\
18.04 & 3 & 149.08 & DMSO \\
23.04 & 3 & 149.07 & DMSO \\
19.06 & 4 & 150.35 & DMSO/difficult to dissolve \\
21.04 & 4 & insoluble (plates) & - \\
26.06 & 4 & insoluble (plates) & - \\
47.18 & 2,3 & 149.17 & DMSO \\
ACN & 2 & 150.09 & DMSO \\
TCI & - & 145.33 & acetone- $d_{6}$ \\
\hline
\end{tabular}

VA-Class: four classes based on the visual pattern presented by the peaks in region 3600-2500 cm-1; NMR: nuclear magnetic resonance; DMSO: dimethylsulfoxide- $d_{6}$; ACN: cyanuric acid; TCI: trichloroisocyanuric acid.

except analyte 19.06, which had a single peak at 150.35 ppm. Analytes 4.10, 205.10, 9.04, 21.04 and 26.06 were insoluble in DMSO (macroscopically, they appeared to be plates, in contrast to the others). Therefore, it can be established that the analyzed samples in solution presented only one peak at $148.58 \pm 0.57 \mathrm{ppm}$. The ${ }^{13} \mathrm{C}$ NMR spectra 
in solution provide a clear indication that the precipitates are symmetric single carbon-type products.

The simulated spectra for cyanuric and isocyanuric acids $^{26}$ showed a peak at $163.87 \mathrm{ppm}$ for the former species and one at $149.90 \mathrm{ppm}$ for the latter species, indicating that isocyanuric acid is the species present in solution and is most likely predominant in the solid product. The value of $150.3 \pm 0.25 \mathrm{ppm}$ is appropriate for the carbon atom of an imide group. The slight displacement of the delta values of most analytes from the central value may indicate a (small) difference in the carbon environment of these analytes.

The spectra of solid ${ }^{13} \mathrm{C}$ NMR for the complete set of analytes in this paper are more complex, and the discussion of these spectra will be presented in another publication jointly with other data.

Furthermore, these measurements allowed the establishment of a clear distinction between VA-classes 1 and 4 and VA-classes 2 and 3 because of their solubility in DMSO: VA-classes 1 and 4 are insoluble (with the exception of analyte 19.06, which was, however, difficult to dissolve), whereas VA-classes 2 and 3 are soluble.

The list is completed with the ${ }^{13} \mathrm{C}$ NMR peaks of Sigma-Aldrich cyanuric acid (150.09 ppm in DMSO) and trichloroisocyanuric acid (145.33 ppm in acetone- $d_{6}$ ).

Additional information can be added here: energy dispersive X-ray spectroscopy (EDS) measurements of analytes of VA-classes 1 and 4 that did not dissolve in DMSO showed the presence of potassium in their structure (analyte 21.04, VA-class 4, 21.6\% K; analyte 26.06, VA-class $4,11.9 \% \mathrm{~K}$; and analyte 205.10 , VA-class 1 , $15.3 \% \mathrm{~K})$. Despite being categorized in VA-class 4 , analyte 19.06 was different from the other analytes of this class: it did not crystallize into plates (unlike all the others) and presented a very low value of potassium: $0.1 \%$. The presence of chlorine, an indication of possible residue from trichloroisocyanuric acid, was detected only in trace amounts in some of the analytes and should not interfere with the spectra of the analytes (the presence of chlorine was verified using very sensitive Feigl tests). ${ }^{23}$

On the one hand, the results from the ${ }^{13} \mathrm{C}$ NMR spectra of a group of analytes in solution described in this paper showed that they had only one peak with practically equal chemical shifts. On the other hand, the bar infrared data of the respective solid analytes differ quite massively. The combination of these two pieces of information suggests the existence of different arrangements of the isocyanuric acid in the solid analytes (polymorphism).

Since all analytes resulting from these reactions were recrystallized from water, presenting a short discussion regarding the observations made of the possible effect of recrystallization on the class of the product formed is pertinent.

In the literature, ${ }^{27}$ crystallization is considered a critical operation regarding polymorphs, where sensitive parameters such as solvent and cooling rate of the solution should be carefully controlled to obtain a given "monomorph". Many references on how to conduct well-monitored crystallization operations, particularly those concerning active pharmaceutical ingredients (APIs), can be found in the literature. ${ }^{28}$

In general, we observed (very small) changes in the number of peaks when the analytes were recrystallized from water but no changes in VA-classes (no strict protocols were used in these recrystallizations). These results lead to a few persistent questions: why does recrystallization of a cyanuric acid analyte of a given VA-class from water tend to produce an analyte of the same class (albeit occasionally adding or subtracting peaks from the spectrum of the original analyte)? Why does the analyte not move toward a more stable configuration? If the solubilization of the analyte would result in the complete disruption of the original solid in isolated molecules (as observed with the dissolution of the analytes in DMSO), then the new solid formed by recrystallization of the dissolved individual molecules would be expected to crystallize into a more stable structure. However, it was observed that even when the crystallizations were conducted under no special conditions, the VA-class of the original analyte was conserved. The retention of the VA-classes after recrystallization of the analytes of different VA-classes suggests that during the process of recrystallization, a "memory" of the original solid form remains in the solution with the entire polymorph or parts of it (oligomorphs) acting as seeds. This proposal also indicates that the solid does not decompose completely in solution to form isolated cyanuric/isocyanuric acid molecules (at least, not under the recrystallization conditions used in this study); otherwise, the migration of at least one of the analytes from a given class to another class would be expected.

Despite these observations, the behavior of some of the analytes causes doubt about the "class conservation" mentioned above. Infrared spectra I001504S and I001504A of analyte 15.04, for instance, are two spectra from the same sample prepared on two different dates, $05 / 07 / 2012$ and $05 / 25 / 2016$, respectively (the only difference between these two spectra seems to be that the analyst picked up different parts of the same sample from the same vial at different times). Table 3 reveals that each spectrum belongs to a different VA-class. According to this Table, the same observation could be made with analytes 17.06 and 47.18. A question could be raised regarding whether these two VA-classes were present 
from the very beginning, i.e., whether they were formed simultaneously during the reaction with TCI, or whether one class moved to the other (albeit only slightly) during the recrystallization process (this possibility could be feasible if some individual molecules of cyanuric acid were formed in the dissolution process, and when cooled, these individual molecules crystallized into another class). Another, more provocative hypothesis is the occurrence of a natural and spontaneous transformation of one class into the other with time and prevalent conditions.

Table 3. The same analytes presenting spectra of different VA-classes

\begin{tabular}{lccc}
\hline Reaction & Analyte & Spectra & VA-class \\
\hline \multirow{2}{*}{15.00} & \multirow{2}{*}{15.04} & $15.04 \mathrm{~B} / \mathrm{S}$ & 3 \\
& & $15.04 \mathrm{~A}$ & 2 \\
\hline \multirow{2}{*}{17.00} & \multirow{2}{*}{17.06} & $17.06 \mathrm{~S} / \mathrm{C}$ & 2 \\
& & $17.06 \mathrm{~A} / \mathrm{B}$ & 3 \\
\hline \multirow{2}{*}{47.00} & \multirow{2}{*}{47.18} & $47.18 \mathrm{~S}$ & 3 \\
& & $47.18 \mathrm{~A} / \mathrm{B} / \mathrm{C}$ & 2 \\
\hline
\end{tabular}

VA-Class: four classes based on the visual pattern presented by the peaks in region $3600-2500 \mathrm{~cm}^{-1}$; spectra ending in $\mathrm{S}$ were made in the period of 2012 and 2013; spectra ending in A, B or C were made in the period of 2015 and 2016.

Another question addresses the crystallization from the mother liquor that resulted from the first crop. Table 4 shows that, in many cases, the second crop belonged to another VA-class, as indicated by its infrared spectra. Analyte 10.04, for instance, presents all three spectra (I001004A, B and C) in VA-class 4, whereas analyte 10.06 exhibited three spectra (I001006A, B and S) in VA-class 3. Reaction 15.00 displays very bizarre behavior: analyte 15.04 exhibits two different VA-classes (I001504A of VA-class 3 and I001504B and S of VA-class 2), whereas all four 15.06 spectra of I001504A have the same VA-class of 3. A hypothesis may also be raised for these facts: a fractionation of the original material when proceeding from one crystallization to the other or a change in class due to the heating and time to which this sample was subjected (less probable). Further research is needed to clarify these occurrences.

\section{Correlation among reaction conditions and VA-class of cyanuric acid formed}

Different observed VA-classes resulting from different reaction conditions raises the question of whether the reaction conditions induce the formation of a certain form of solid cyanuric acid (VA-class). In addition to the theoretical interest in understanding how the conditions may induce the structure of the solid formed, having
Table 4. Different analytes of a same reaction showing different VA-classes

\begin{tabular}{lccc}
\hline Reaction & Analyte & Spectra & VA-class \\
\hline \multirow{2}{*}{1.00} & 9.04 & I000904A/B/C/S & 1 \\
& 9.06 & I000906S & 2 \\
\hline \multirow{2}{*}{10.00} & 10.04 & I001004A/B/C & 4 \\
& 10.06 & I001006A/B/S & 3 \\
\hline \multirow{4}{*}{15.00} & 15.04 & I001504A & 3 \\
& 15.04 & I001504B/S & 2 \\
& 15.06 & I001506A/B/C/S & 3 \\
\hline \multirow{4}{*}{18.00} & 17.05 & I001705A/B/C/S & 2 \\
& 17.06 & I001706A/B & 3 \\
& 17.10 & I001706C/S & 2 \\
& 17.18 & I001710 & 2 \\
& 18.04 & I001804A/B/C/S & 3 \\
\hline \multirow{3}{*}{33.00} & 18.06 & I001806S & 3 \\
& 18.09 & I001809S & 4 \\
\hline \multirow{4}{*}{ VA } & 33.04 & I003304S & 3 \\
& 33.09 & I003309S/A/B/C/D/E & 3 \\
& 33.11 & I003311S/A/B/C & 3 \\
\hline
\end{tabular}

VA-Class: four classes based on the visual pattern presented by the peaks in region 3600-2500 $\mathrm{cm}^{-1}$; spectra ending in $\mathrm{S}$ were made in the period of 2012 and 2013; spectra ending in A, B or C were made in the period of 2015 and 2016.

procedures able to reproduce a given class for further studies would also be useful.

An overall view of the reaction conditions and the VA-classes produced is presented in Supplementary Information Table S1, which shows that 135 analytes belong to VA-class 1, 53 to VA-class 2, 29 to VA-class 3 and 27 to VA-class 4 . Sixteen analytes were derived from Sigma-Aldrich cyanuric acid (VA-class Y), bringing the total to 27 different reaction conditions and 151 analytes and the corresponding infrared bar spectra. These analytes include the different crystallization fractions of the product of a reaction and the repetitive infrared spectra obtained for each of the analytes.

Four variables were studied in these reactions: $(i)$ the nature of the salt (potassium bromide and chloride, ammonium chloride and bromide and no salt); (ii) the temperature of the reaction. As the reaction is quite exothermic, particularly when ammonia is used, the temperature was carefully controlled by performing some reactions an Easy-Max reactor (Mettler-Toledo), and these reactions were compared with reactions performed in normal laboratory flasks with little attention paid to temperature changes. As TCI was added via the slow and dropwise introduction of a solution of the compound in 
acetone (with the exception of reaction 31.00, in which small lumps of solid TCI were added directly to the ammonia solution), the temperature of the reaction flask did not change much; (iii) the nature of the medium, which was varied from water/acetone, ammonia/acetone, dilute ammonia/acetone and pure ammonia; and (iv) the presence or absence of methyl acrylate or the use of acrylonitrile as a substitute for the acrylate as the chlorine/bromine acceptor.

The data in Table S1 were used to produce Table 5, which lists 19 different reaction conditions that lead to analytes of only one VA-class.

A more detailed analysis of the influence of the reaction parameters used to define which reactions lead to a certain VA-class will now be presented:

Table 5 shows that the formation of VA-class 1 is strongly dependent on the presence of potassium salts and that no VA-class 1 analytes are formed when ammonium salts or no salt are used. Conversely, VA-class 2 and VA-class 3 analytes occur mainly when ammonium salts are used. VA-classes 1 and 4 occurs mainly when $\mathrm{KBr}$ is used. The 21 reactions performed with $\mathrm{KCl}$ are distributed among VA-classes 1 (10), 2 (8) and 3 (3), whereas no VA-class 4 analytes were produced.

Regarding the influence of temperature, Table 5 shows that lower temperatures $\left(0{ }^{\circ} \mathrm{C}\right)$ favor the formation of VA-classes 3 and 4 over VA-classes 1 and 2. The reaction is highly exothermic, and the number of reactions that lead to VA-class 2 analytes is greater when the temperature is increased from 0 to $25^{\circ} \mathrm{C}$ and even when the reactions are conducted without strict temperature control (r.t./ca. 28+). These findings indicate that the formation of VA-class 2 products could be favored by an increase in temperature.

The products of the reactions performed in $\mathrm{H}_{2} \mathrm{O} /$ acetone and $\mathrm{NH}_{4} \mathrm{OH} /$ acetone are not neatly distributed

Table 5. Reactions leading to analytes of a single VA-class

\begin{tabular}{|c|c|c|c|c|c|c|c|}
\hline \multicolumn{2}{|c|}{ Halogen acceptor } & \multirow{2}{*}{$\frac{\text { TCI }}{\text { No. of moles }}$} & \multicolumn{2}{|c|}{ Salt } & \multirow{2}{*}{$\begin{array}{c}\text { Temperature / } \\
{ }^{\circ} \mathrm{C}\end{array}$} & \multirow{2}{*}{ Medium/solvent } & \multirow{2}{*}{ Reaction } \\
\hline Acceptor & No. of moles & & Salt & No. of moles & & & \\
\hline \multicolumn{8}{|c|}{ VA-class 1} \\
\hline AcrMet & 0.056 & 0.020 & $\mathrm{KBr}$ & 0.116 & r.t./ca. $28+$ & $\mathrm{H}_{2} \mathrm{O}$ /acetone & 2.00 \\
\hline AcrMet & 0.050 & 0.017 & $\mathrm{KBr}$ & 0.100 & r.t./ca. 28+ & $\mathrm{H}_{2} \mathrm{O}$ /acetone & $\begin{array}{c}3.00 \\
4.00 \\
205.00\end{array}$ \\
\hline- & 0.000 & 0.017 & $\mathrm{KBr}$ & 0.116 & r.t./ca. $28+$ & $\mathrm{NH}_{4} \mathrm{OH} /$ acetone & 11.00 \\
\hline \multicolumn{8}{|c|}{ VA-class 2} \\
\hline- & 0.000 & 0.017 & - & 0.000 & r.t./ca. $28+$ & $\mathrm{NH}_{4} \mathrm{OH} /$ acetone & 12.00 \\
\hline AcrMet & 0.050 & 0.017 & $\mathrm{NH}_{4} \mathrm{Br}$ & 0.100 & r.t./ca. $28+$ & $\mathrm{H}_{2} \mathrm{O} /$ acetone & 14.00 \\
\hline AcrMet & 0.111 & 0.371 & $\mathrm{NH}_{4} \mathrm{Cl}$ & 0.222 & 25 & $\mathrm{H}_{2} \mathrm{O} /$ acetone & 29.00 \\
\hline AcrMet & 0.050 & 0.017 & - & 0.000 & r.t./ca. $28+$ & $\mathrm{HCl} / \mathrm{H}_{2} \mathrm{O} /$ acetone & 210.00 \\
\hline AcrNit & 0.050 & 0.017 & $\mathrm{KCl}$ & 0.100 & r.t./ca. $28+$ & $\mathrm{H}_{2} \mathrm{O} /$ acetone & 212.00 \\
\hline AcrMet & 0.050 & 0.017 & $\mathrm{KCl}$ & 0.100 & r.t./ca. $28+$ & $\mathrm{H}_{2} \mathrm{O}$ /acetone & 214.00 \\
\hline AcrNit & 0.050 & 0.017 & $\mathrm{KCl}$ & 0.100 & r.t./ca. $28+$ & $\mathrm{H}_{2} \mathrm{O}$ /acetone & 215.00 \\
\hline \multicolumn{8}{|c|}{ VA-class 3} \\
\hline - & 0 & 0.017 & - & 0.000 & -10 & $\mathrm{NH}_{4} \mathrm{OH}_{\mathrm{aq}} /$ acetone & 24.00 \\
\hline- & 0 & 0.017 & - & 0.000 & r.t./ca. $28+$ & $\mathrm{NH}_{4} \mathrm{OH}_{\mathrm{aq}} /$ acetone & 25.00 \\
\hline AcrMet & 0.05 & 0.017 & $\mathrm{KCl}$ & 0.100 & 25 & $\mathrm{H}_{2} \mathrm{O}$ /acetone & 27.00 \\
\hline \multicolumn{8}{|c|}{ VA-class 4} \\
\hline AcrMet & 0.050 & 0.017 & $\mathrm{KBr}$ & 0.012 & 0 & $\mathrm{H}_{2} \mathrm{O} /$ acetone & 20.00 \\
\hline AcrMet & 0.050 & 0.017 & $\mathrm{NH}_{4} \mathrm{Br}$ & 0.100 & r.t./ca. $28+$ & $\mathrm{NH}_{4} \mathrm{OH} /$ acetone & 16.00 \\
\hline AcrMet & 0.050 & 0.017 & $\mathrm{NH}_{4} \mathrm{Br}$ & 0.100 & 0 & $\mathrm{NH}_{4} \mathrm{OH}_{\mathrm{aq}}$ /acetone & 19.00 \\
\hline AcrMet & 0.050 & 0.017 & $\mathrm{KBr}$ & 0.116 & 0 & $\mathrm{NH}_{4} \mathrm{OH}_{\mathrm{aq}}$ /acetone & 21.00 \\
\hline AcrMet & 0.050 & 0.017 & $\mathrm{KBr}$ & 0.116 & 25 & $\mathrm{H}_{2} \mathrm{O}$ /acetone & 26.00 \\
\hline- & 0.000 & 0.017 & - & 0.000 & -15 & $\mathrm{NH}_{4} \mathrm{OH}$ & 31.00 \\
\hline
\end{tabular}

TCI: trichloroisocyanuric acid; VA-class: four classes based on the visual pattern presented by the peaks in region $3600-2500 \mathrm{~cm}^{-1}$; AcrMet: methyl acrylate; AcrNit: acrylonitrile; r.t.: room temperature. 
among VA-classes 1,3 and 4, although it seems that $\mathrm{H}_{2} \mathrm{O}$ /acetone favors VA-class 1 slightly more. However, this latter medium seems to very strongly induce the formation of VA-class 2 analytes.

To determine the role of the acrylate moiety in VA-class formation, reactions were performed with no methyl acrylate or acrylonitrile and compared with those conducted with these chlorine/bromine acceptors. The reactions of TCI dissolved in acetone with ammonia/ acetone and with or without salt at different temperatures produced all types of VA-classes. However, only VA-class 4 was produced when the reaction was performed with solid TCI on pure ammonia (no acetone), no acrylate moiety and no salt at $-15{ }^{\circ} \mathrm{C}$. The reaction of solid TCI with ammonia is very exothermic. At room temperature, it produces plenty of fumes of ammonium chloride and, after a few minutes, starts producing a strong green color upon reacting with the Berthelot reagent. ${ }^{29}$ This observation can be explained by the formation of chloramine $\left(\mathrm{NH}_{2} \mathrm{Cl}\right)$ and its involvement as an intermediate in the process of color production because, as stated by Patton and Crouch, ${ }^{30}$ "when sodium aquopentacyanoferrate was substituted for sodium nitroprusside the reaction mixture turned green". No green color being produced when the reaction is conducted with TCI dissolved in acetone and further treated with the Berthelot reagent (only a brown product is formed) may be a good indication of chloramine formation since, in acetone, it would be captured to form a Schiff base. Studies are in progress to clarify these observations.

We may conclude this section by stating that analytes of a single VA-class may be produced when the reaction conditions presented in Table 5 are followed. Nevertheless, although no strict controlled conditions for the reactions were used, it should be stressed that if there is an objective to obtain a certain VA-class, it is advisable to conduct the reactions under stringently controlled conditions, particularly those involved in the crystallization processes. ${ }^{27}$

The bar infrared spectra of solid cyanuric acid analytes: maps of analyte frequency distribution and classes of cyanuric acid analytes

The diversity of frequencies presented by the infrared spectra of the analytes obtained from the reactions with trichloroisocyanuric is, indeed, overwhelming. This section is a tentative attempt to find some order in the chaos of frequencies encountered.

The first step to achieve this goal was to confine analytes into classes based on similar patterns presented (visually) in region A' (3600-2500 $\left.\mathrm{cm}^{-1}\right)$ of the infrared spectrum. Four classes were defined, called VA-classes 1 to 4 , as presented in the beginning of this session.

FI-plots very conveniently allow the recognition of similarities among the analytes (after all, they are all cyanuric acid), as well as differences among the classes or among analytes of a given class. Moreover, these plots very clearly show the great diversity of the infrared absorption frequencies of the analytes.

Figure 5 displays the FI-plot for the cyanuric acid VA-classes, showing the distribution of the frequencies for the various classes. The most notable feature of this plot is that the distribution of the frequencies is different for each VA-class and that no two classes are superimposable. Although they show common frequencies, these classes concurrently present characteristic frequencies not present in the others. This latter feature was the criterion used to define a VA-class. The FI-plot makes the differences among the classes very clear and precise.

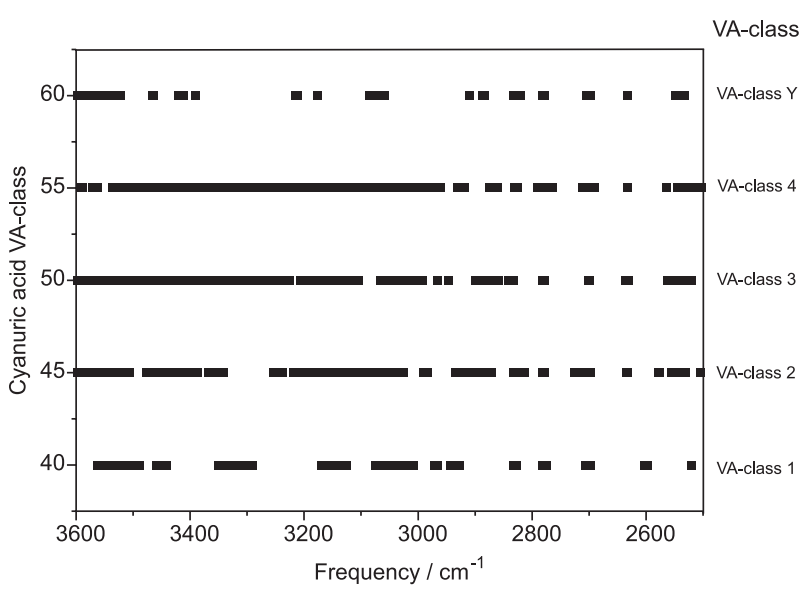

Figure 5. FI-plot for cyanuric acid VA-classes.

Figures 6-10 show FI-plots for the analytes of VA-classes 1 to 4 and for the Sigma-Aldrich cyanuric acid. Because a class defines a proper pattern for the structure of the solids, the members of a class would be expected to exhibit very similar spectra. However, although they present many common peaks at a first glance, the analytes of a single class are observed to not all absorb exactly at the same frequencies, with the differences mainly being due to the diversity of the structures of each solid analyte (it should also be considered the error of the infrared instrument). Figures 6-10 show that they actually present similarities, i.e., many frequencies are common to all analytes of the class, but they also present many differences, i.e., many frequencies do not have similarities among all the analytes. The SigmaAldrich cyanuric acid spectra (VA-class Y) are remarkably similar, but not equal. Through the bar representation of the spectra, these graphs very clearly stress once more the 


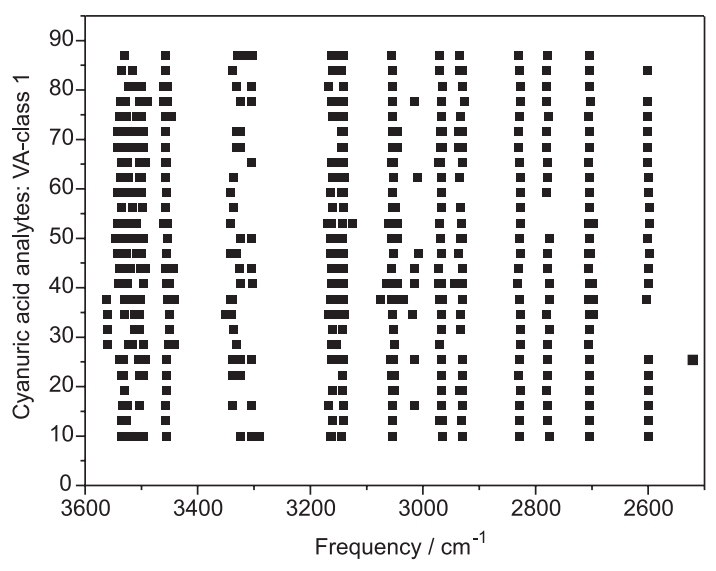

Figure 6. FI-plot for VA-class 1 analytes.

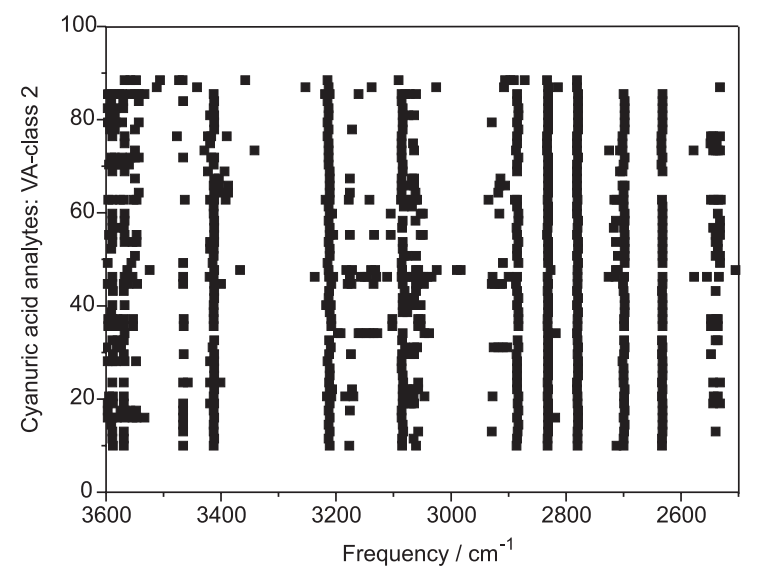

Figure 7. FI-plot for VA-class 2 analytes.

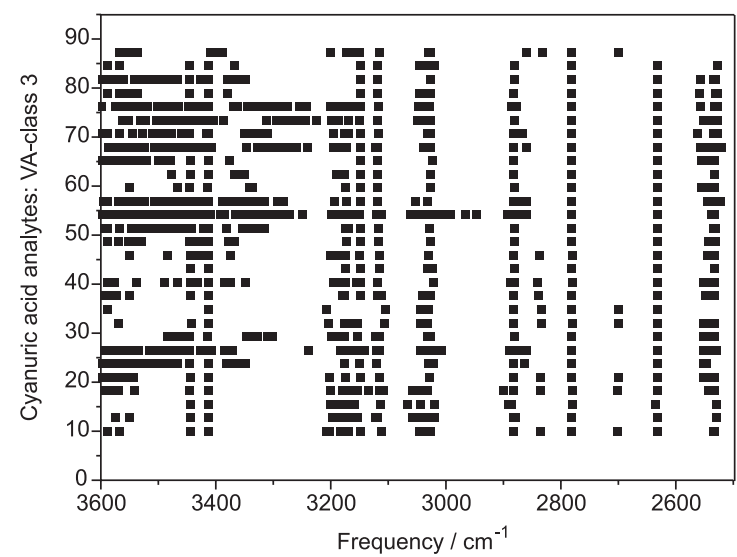

Figure 8. FI-plot for VA-class 3 analytes.

great diversity of infrared absorption frequencies for solid cyanuric acid and, consequently, the great diversity of spatial arrangements that solid cyanuric acid may assume.

The class marker concept

The next step in the analysis of these spectra raises the concept of class markers, which are defined as a

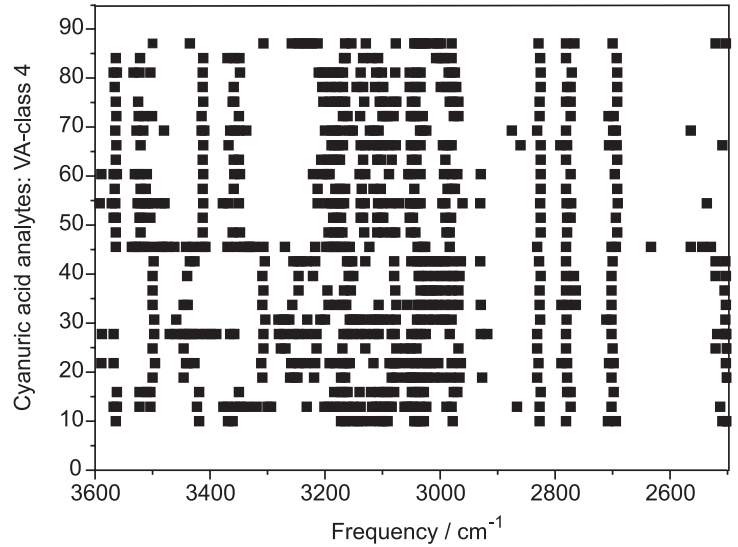

Figure 9. FI-plot for VA-class 4 analytes.

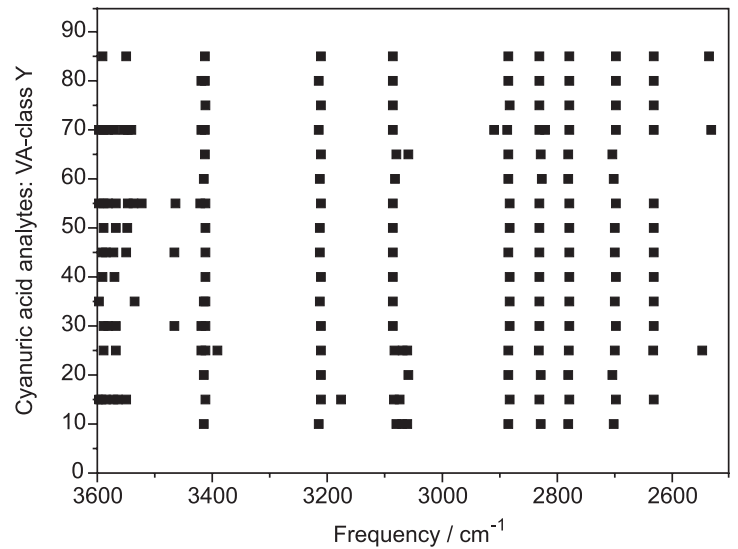

Figure 10. FI-plot for VA-class $Y$ analytes.

characteristic set of frequencies of a VA-class that will not collect analytes from another class when applied to a mixture.

To find frequencies that could be used to define a class, a group of frequencies must be used. A group of five frequencies was then chosen to define, for each of the five classes, arrays of five characteristic frequencies of each VA-class that, at the same time, do not collect analytes of any other class.

To determine the class markers, arrays of 5 frequencies must be computed for each VA-class by combining the (different) frequencies of the analytes of that class with size 5 and then searching for which analytes absorb in all and each of the generated 5 frequencies (plus or minus the error considered). However, using all the different frequency values of a VA-class would require the handling of a number of possible arrays that may be too high for the software/computer presently in use (for instance, combining VA-class 1 containing 146 different frequencies with size 5 leads to $15,853,624$ combinations).

Table 6 presents 5 -frequency markers for VA-classes 1 to 4 and for the Sigma-Aldrich cyanuric acid class, covering the region A' of the infrared spectrum. The total number 
Table 6. 5-Frequency markers for cyanuric acid VA-classes

\begin{tabular}{|c|c|c|c|c|c|c|c|c|c|}
\hline \multirow{2}{*}{ Class/region } & \multirow{2}{*}{$\begin{array}{l}\text { No. of analytes in the } \\
\text { class }\end{array}$} & \multirow{2}{*}{$\begin{array}{l}\text { No. of analytes in the } \\
5 \text {-frequencies marker }\end{array}$} & \multirow{2}{*}{$\delta$} & & \multicolumn{5}{|c|}{ 5-Frequency marker $/ \mathrm{cm}^{-1}$} \\
\hline & & & & & Frequency 1 & Frequency 2 & Frequency 3 & Frequency 4 & Frequency 5 \\
\hline \multirow{2}{*}{$\mathrm{VA}^{\mathrm{a}}$} & \multirow{2}{*}{26} & 22 & 4 & a & 3144 & 3055 & 2968 & 2829 & 2704 \\
\hline & & 22 & 4 & $\mathrm{~b}$ & 3456 & 3143 & 3055 & 2829 & 2704 \\
\hline \multirow{2}{*}{ VA2 } & \multirow{2}{*}{53} & 46 & 7 & a & 2885 & 2832 & 2780 & 2700 & 2633 \\
\hline & & 36 & 17 & $\mathrm{~b}$ & 3213 & 3084 & 2885 & 2831 & 2780 \\
\hline \multirow{2}{*}{ VA3 } & \multirow{2}{*}{29} & 23 & 6 & $\mathrm{a}$ & 3446 & 3413 & 2881 & 2779 & 2631 \\
\hline & & 18 & 11 & $\mathrm{~b}$ & 3446 & 3149 & 3028 & 2882 & 2779 \\
\hline \multirow{2}{*}{ VA4 } & \multirow{2}{*}{27} & 12 & 15 & $\mathrm{a}$ & 3035 & 3032 & 2981 & 2978 & 2827 \\
\hline & & 8 & 19 & $\mathrm{~b}$ & 3564 & 3167 & 3043 & 2978 & 2827 \\
\hline \multirow{2}{*}{ VAY $^{b}$} & \multirow{2}{*}{16} & 15 & 1 & $\mathrm{a}$ & 3413 & 3213 & 2885 & 2829 & 2779 \\
\hline & & 13 & 3 & $\mathrm{~b}$ & 3213 & 3084 & 2885 & 2781 & 2700 \\
\hline
\end{tabular}

aVA1 stands for visual spectrum of class 1 for the region A' of the infrared spectrum, and so on; 'the letter "Y" in VAY refers to the class/group of cyanuric acid samples derived from the Sigma-Aldrich product. $\delta$ : difference between analytes in the class and analytes in the 5-frequencies marker; a: the marker that contains the maximum number of analytes among all 5-frequency markers; b: the marker that contains all important/reference peaks of the printed spectra. Region A': $3600-2500 \mathrm{~cm}^{-1}$ (error $\left.= \pm 2 \mathrm{~cm}^{-1}\right)$.

of peaks of the class and the number of peaks of the corresponding marker are also shown.

Two types of markers are shown in Table 6: one (frequencies present in lines marked "a") that collects the largest number of analytes, sometimes close to the total number of analytes of the VA-class, and the other (shown in lines "b") that contains all important/reference peaks of the printed spectra. Markers of line "a" may not collect some of the peaks that were used visually to define the VA-class because only a limited number of groups of 5 frequencies derived from the total number of frequencies of the class were computed. Therefore, a question arises: which marker should be preferred for representing the VA-classes when needed? The markers with the largest number of analytes, or the others encompassing fewer analytes but containing the frequencies responsible for the visual classification of the VA-classes? It seems more reasonable to adopt the marker that collects the largest number of analytes as the one to use as the VA-class marker when needed. In this way, the VA-class of any cyanuric acid analyte may be determined by determining the continence (instead of the coincidence) of the respective spectra with the V-class markers. This tool may be of particular importance when applied to the characterization of API polymorphs.

Figures 11 and 12 show FI-plots for these two types of 5 -frequency markers in order to emphasize that diversity is always present.

Coincident frequencies among the VA-classes indicate that the corresponding analytes should contain similar structural moieties in the same way that non-coincident frequencies should correspond to different structural

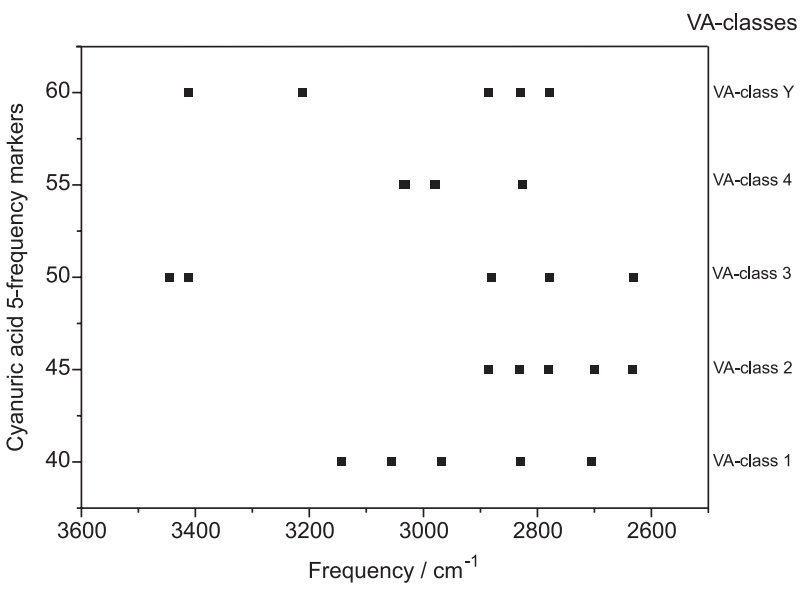

Figure 11. FI-plot of 5-frequency markers with the largest number of analytes (lines marked "a" in Table 6).

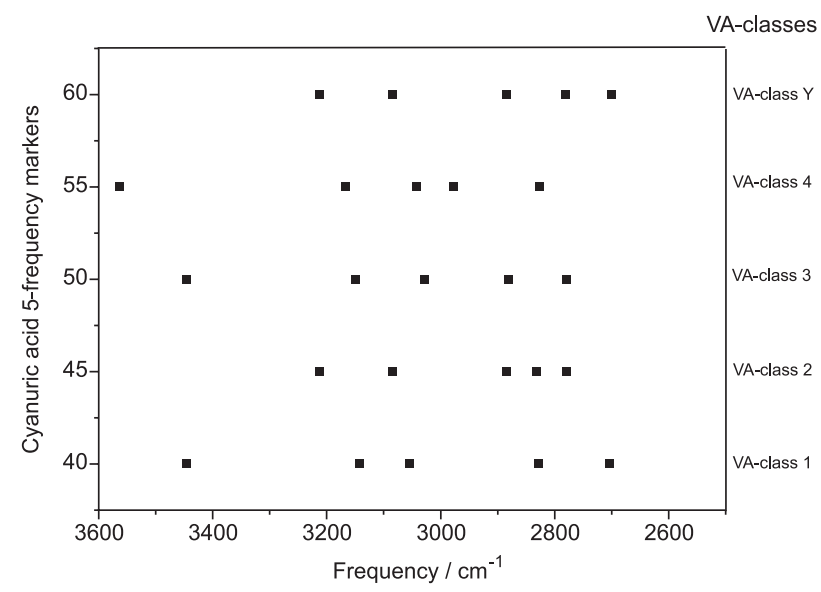

Figure 12. FI-plot of 5 -frequency markers containing the most significant peaks shown in the "printed" spectra (lines marked "b" in Table 6). 
moieties. Both sets of information are important for establishing the 3D structures for the solids.

These results show that although the initial choice of the VA-classes was made empirically (visually), it is possible to recognize sets of characteristic frequencies of analytes of each VA-class that are absent in the others, thereby providing evidence for the initial hypothesis of the existence of these classes. The common peaks among the classes define cyanuric acid as a chemical species, whereas the characteristic group of peaks of a class establishes that the analyte belongs to a particular set of structures of solid cyanuric acid.

The coincidence and continence indexes for the cyanuric acid analytes

A very general analysis of the infrared spectra of cyanuric acid involves the calculation of the respective coincidence and continence indexes among all the analytes of the groups. This calculation is accomplished using confrontation matrices. In the present case, a $159 \times 159$ confrontation matrix was set to calculate both the SCI relative to the reference (which indicates how much of the reference is contained in the analyte) and the DCI (which provides some more information about the coincidence between the spectra) for the 159 spectra of the analytes of cyanuric acid analyzed in this paper. The resulting 2D matrix has 25077 slots filled with the respective indexes. For the purpose of analysis, it is better to have the results presented in a list sorted in descending order of the double and single coincidence indexes. However, in any case, the list is too long to be included in this text; thus, only the relevant results will be shown and discussed here. For more details about these Tables/databases, the authors should be contacted.

Table S1 (Supplementary Information section) also allows a discussion regarding the issue of the analytes that have a $100 \%$ coincidence index (equal analytes) or a $0 \%$ coincidence index (completely different analytes). Figure 13 shows these cases. A case of $100 \%$ coincidence may be expected for analytes from the same reaction (as occurs with analytes 3.10.A and 3.10.C of VA-class 1); however, pairs of spectra of analytes from different reactions with $100 \%$ coincidence were also observed: 2804C and 3311C, both of VA-class 2.

In contrast to the cases with $100 \%$ coincidence, in the pairs with $0 \%$ coincidence, each analyte belongs to a different VA-class, but no pairs with analytes of VA-class 4 were formed.

In the cases where the single coincidence index is higher than that of double coincidence index, the number of peaks of the analyte is generally much higher than the number of peaks of the reference, indicating that the reference is completely contained within the analyte and that the analyte has some peaks "left over". For instance, the single continence index between analytes $2904 \mathrm{~S}$ (VA-class 2, 36 peaks) and 1204S (VA-class 2, 11 peaks) is $100 \%$, whereas the double coincidence index is $48.94 \%$. However, there are some cases where the single continence index is lower than the double coincident index, as observed with the pair 4718B (VA-class 2, 13 peaks) and 3309A (VA-class 2, 14 peaks) having a double coincidence index of $62.96 \%$ and single continence index of $57.14 \%$. In this
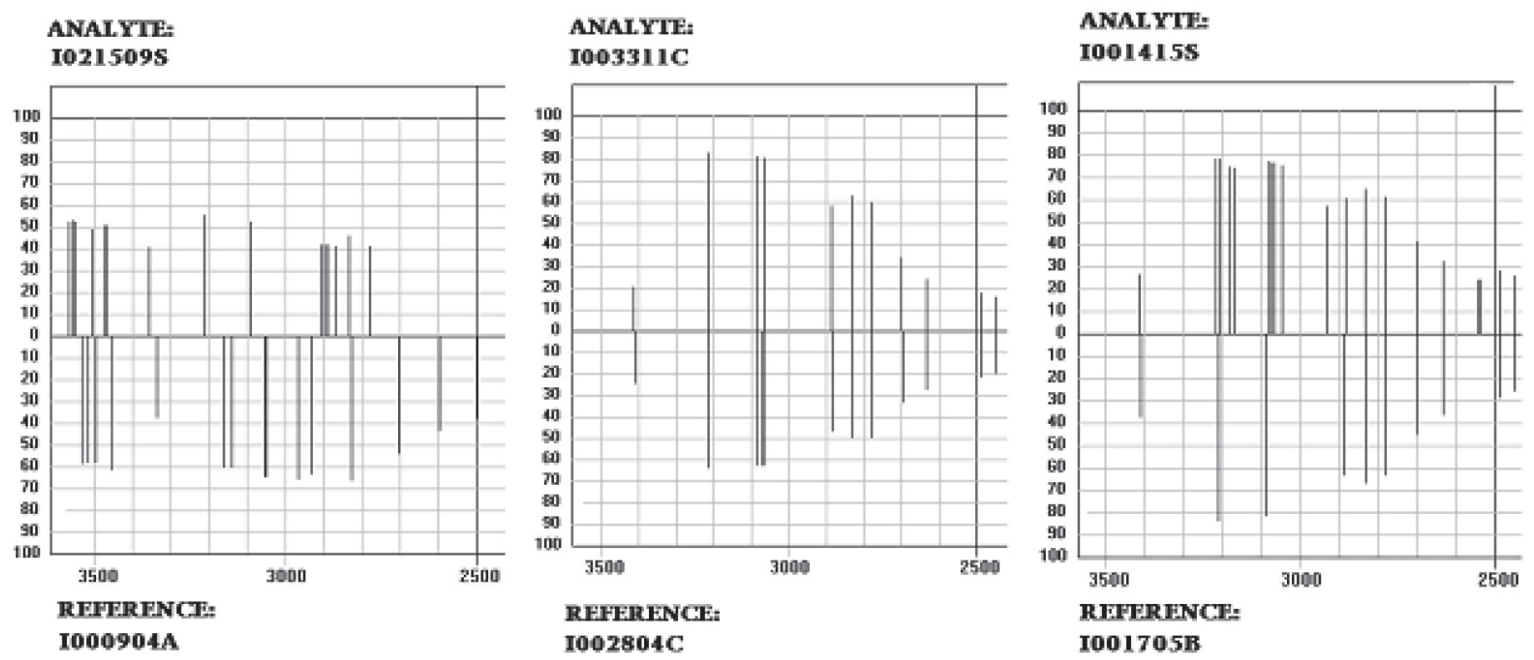

Figure 13. Graphic comparison of analytes according to their coverage indexes: Left: two analytes with a $0 \%$ double coincidence index (no coincidence exists among any of the peaks of analytes I021509S and I000904A). Middle: two analytes with a 100\% double coincidence index (all peaks of analytes I003311C and I002804C coincide). Right: two analytes with 57.15\% double coincidence index and 100\% single continence index of the reference (I001705B) relative to the analyte (I001415S). Notice that the reference is completely contained within the analyte. Notice also that in an enlarged view of the spectra, not all the peaks of the reference coincide perfectly with those of the analyte, but the software takes them as coincident due to the error of $\pm 2 \mathrm{~cm}^{-1}$ given to the frequency values. 

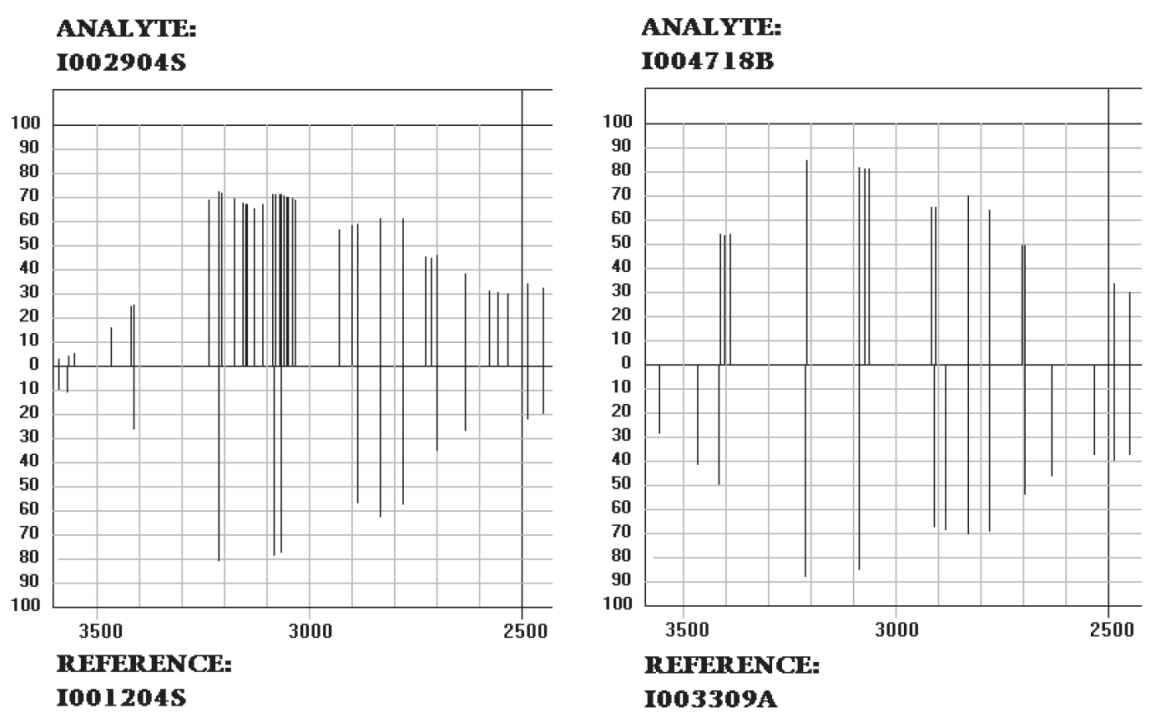

Figure 14. Graphic comparison of analytes $2904 \mathrm{~S}$ and 20919S presenting a single coincidence index of $100 \%$ and a double continence index of $42.22 \%$ and of analytes $4718 \mathrm{~B}$ and $3309 \mathrm{~A}$ presenting a double coincidence index of $62.96 \%$ and a single coincidence index of $57.14 \%$.

case, only ca. half of the peaks of the reference coincide with peaks of the analyte. Thus, there is a reasonable "left over" from both analytes. The spectra relative to these two cases are shown in Figure 14.

Analytes selected from a 5-frequency marker generally have high double coincidence and single continence indexes, as expected. For example, the pair $1706 \mathrm{~S}$ and 3309S exhibits a double coincidence index of $96.30 \%$ and a single coincidence index of $92.86 \%$. However, this does not occur in all cases. For instance, with the pair 21218B and $3309 \mathrm{~A}$, the double coincidence index is $61.54 \%$, and the single coincidence index is $57.14 \%$. Therefore, although some patterns could be established for the solid cyanuric acid analytes, the diversity of structures is high, and a spreading of all the parameters can be observed over the whole range of frequencies.

The results of a small study of the coincidence and continence of the Spectral Database for Organic Compounds $\left(\right.$ SDBS) ${ }^{31}$ spectrum for cyanuric acid (No. 2043) and the common spectra for the Sigma-Aldrich cyanuric acid are particularly interesting. A common spectrum is, as the name indicates, a spectrum that results from a comparison operation of different spectra of a group, leaving only the peaks that are common to the various spectra of the group. It is used primarily to "average" (to some extent) the slightly different spectra of an analyte into one spectrum.

Table 7 clearly shows the differences between these spectra based on their coverage indexes: a double coincidence index of only $26.19 \%$, and the continence of the SDBS spectra into the Sigma-Aldrich spectra of $22.73 \%$.

The bar graph representations of these two spectra are also compared (Figure 15), again showing very clearly the difference between these spectra.

Use of coincidence indexes to measure the homogeneity of an analyte

Coincidence and continence indexes are good qualitative and quantitative criteria for defining the similarity and difference among bar spectra. In this way, the use of these indexes can be extended further to measure the homogeneity of an analyte. Previously, the set of spectra of a solid analyte taken from a same vial at different times was shown to often be different from one another (some are only slightly different, but very rarely they are all equal), with the difference arising from heterogeneities in the

Table 7. Comparison of the coverage indexes between the Sigma-Aldrich and SDBS cyanuric acid infrared spectra. Region: $3500-500 \mathrm{~cm}^{-1}$; intensity: $0-100$; error $= \pm 2 \mathrm{~cm}^{-1}$

\begin{tabular}{|c|c|c|c|c|c|c|c|}
\hline \multicolumn{2}{|c|}{ Sigma-Aldrich ACN (analyte) } & \multirow[b]{2}{*}{$\begin{array}{c}\text { No. of } \\
\text { coincident peaks }\end{array}$} & \multicolumn{2}{|c|}{ SDBS (reference) } & \multicolumn{3}{|c|}{ Coverage index $/ \%$} \\
\hline No. of peaks & $\begin{array}{c}\text { No. of } \\
\text { non-coincidence } \\
\text { peaks }\end{array}$ & & $\begin{array}{c}\text { No. of } \\
\text { non-coincidence } \\
\text { peaks }\end{array}$ & No. of peaks & Double & Single (reference) & Single (analyte) \\
\hline 22 & 17 & $5 / 6$ & 14 & 20 & 26.19 & 30.00 & 22.73 \\
\hline
\end{tabular}

ACN: cyanuric acid; SDBS: Spectral Database for Organic Compounds. 


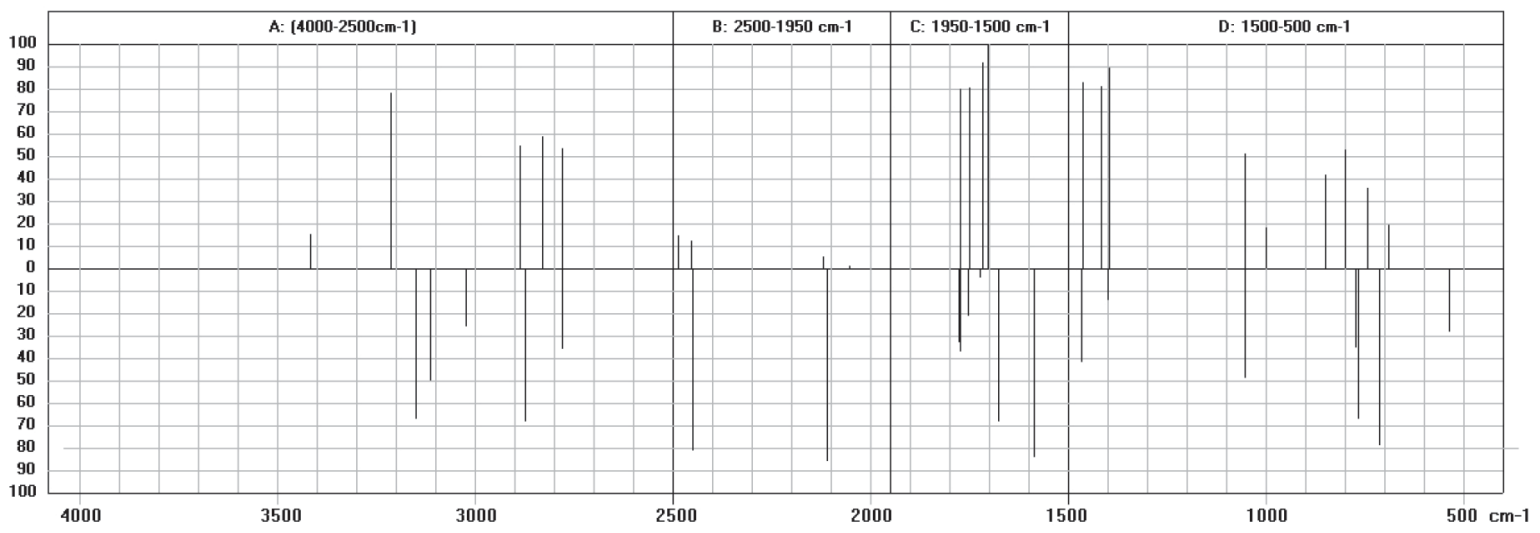

Figure 15. Comparison between the cyanuric acid spectra from Sigma-Aldrich (upper spectrum) and SDBS (lower spectrum).

sample. The concept of coincidence indexes may thus be extended to quantify the homogeneity of an analyte via a homogeneity index (HI) calculated from the coincidences among the samples. To calculate this index, it is first necessary to calculate the double coincidence indexes among the analytes using a confrontation matrix among the bar spectra of the analytes involved in the determination of the homogeneity. Second, the calculated values of the coincidence indexes are averaged, and the mean deviation is also calculated. The $\mathrm{HI}$ is then calculated by means of the following equation:

Homogeneity index $=$ average $-(\delta \times$ mean deviation $)$

This equation indicates that homogeneity increases when the average increases (in other words, when the coincidence among the analytes increases). However, the average does not account for everything.

Adding the concept of dispersion to the values around the average is necessary because homogeneity increases when the dispersion of values around the average decreases. Therefore, the mean deviation should also be used to complement the calculation of homogeneity. $\delta$ is an empirical factor (chosen by the analyst) that may be added to the calculations to modulate the contribution of the mean deviation: it may vary from 0 (neglecting the influence of the deviations) upward. One is considered the default value.

To demonstrate the use of the $\mathrm{HI}$, an example involving the group of analytes 0310A, 0310B, 0310C and 0310S follows. Four spectra of these analyte generated 12 coincidence indexes calculated from the respective square matrix of $4 \times 4$ (confrontation between equal spectra are eliminated). The average value of the indexes is 81.93 , and the mean deviation is 6.02 . For a value of $\delta=1$, the homogeneity indexes will be 75.91 relative to the double coincidence index. This indicates that the homogeneity of an analyte can now be associated with a number calculated from coincidences among their bar infrared spectra. Homogeneity can be scaled considering that for a completely homogeneous sample $\mathrm{HI}=100$ (equal samples will give a coincidence indexes equal to 100), the average will also be 100 , and the mean deviation is zero for any value of $\delta$. If the analyte comprises a mixture of completely different analytes (no coincidences at all among the spectra), then $\mathrm{HI}=0$. Mixtures of solid analytes of the same species will give intermediate values between 0 and 100: the more homogeneous the sample, the closer the HI will be to 100 .

All analytes described in this paper are solids composed of one species of a molecule: isocyanuric acid (although some analytes may contain some of its tautomer, cyanuric acid). If the definition of polymorph is derived directly from what the word says (many forms, from the Greek poly $=$ many and morph $=$ form), then the special "macro" shapes that these analytes show (plates, rods, sometimes not well-defined agglomerates of "particles", a fine talc-like powder, etc.) give the observer the impression that they should be seen as polymorphs, as indicated by their quite different infrared spectra. We can therefore say that we have obtained polymorphs of cyanuric acid. However, due to the great diversity of infrared spectra that these analytes present, it is difficult to say how proper it would be to define which are the crystals of cyanuric acid and/or polymorphs because it is difficult to compare the observed properties with the ones presently required to characterize polymorphs. ${ }^{27}$ Notably, none of the cyanuric acid analytes melt but instead sublime.

Polymorphs are generally characterized more thoroughly by a group of analytical methods, including infrared spectra, X-ray diffraction, thermal analysis and optical microscopy. ${ }^{27}$ We decided to limit this paper solely to a discussion of the method that uses the infrared bar spectra, with its maps of frequencies and coincidence indexes, as a precondition for develop the models necessary to better 
explain the vibrational behavior of solid cyanuric acid. The coupling of infrared bar results with X-ray diffraction, thermal analysis and microscopy of the samples from this paper will be the subject of another publication, which will aim to more rigorously define the solid phase arrangements of cyanuric/isocyanuric acid molecules.

\section{Conclusions}

This paper showed the great diversity of the infrared spectra of the analytes produced from various reactions of trichloroisocyanuric acid (all said to be cyanuric acid). It also described the characterization of the similarities and differences among the products through the use of graphs (FI-plots) or numbers (indexes), both of which were calculated from the respective bar spectra.

The method used in this paper to create an infrared bar spectra directly from the output of the spectrometer was successful, and the produced bar spectra could be used to quantitatively compare infrared spectra via coincidence/ continence indexes. The usefulness of the method was demonstrated with a set of infrared bar spectra of the white precipitates resulting from reactions of trichloroisocyanuric acid, all of which were said to be cyanuric acid. Using this method enabled the demonstration of the great diversity of the cyanuric acid analyte spectra, which could be grouped into (mainly) four classes (VA-classes). The ability to group these spectra suggests the presence of polymorphs.

The simple three consecutive points algorithm was effective in creating a bar spectrum directly from the data generated by the spectrometer. In addition, the common peaks algorithm can be effectively used to calculate coincidence and continence indexes in confrontation matrices. Furthermore, any type of comparison and calculation is readily accomplished using bar spectra due to the ease with which they can be handled by software.

Class markers, a set of five bar frequencies common to the analytes of a VA-class, were established for each VA-class of cyanuric acid analytes. The concepts of both class and class markers may be used to characterize classes of analytes, including polymorphs.

Many applications can be ascribed to coincidence and continence indexes based on the bar infrared spectra of analytes, as it was shown to define, quantitatively, differences among analytes. The use of coincidence and continence indexes can be further extended to calculate the homogeneity of an analyte, to clustering sets of different analytes (in development), etc.

An important contribution that this study offers is showing how important a bar spectrum could be to define the structure of solid analytes because of its ability to display precise frequencies in a simple discrete display, facilitating the production of algorithms and design software to answer posed questions. Calculations of coincidence indexes and the establishment of class markers and FI-plots are examples of the ability of bar spectra to translate spectra into numbers.

The data provided in this paper indicate the need for a deeper study to correlate frequencies to molecular moieties in solid cyanuric/isocyanuric acid and for better models to simulate vibration frequencies in order to describe the configurations of molecules in space that mimic those of the bar spectra of real molecules.

Finally, the use of the bar spectra together with coincidence indexes may be seen as a step forward for infrared spectrometry because it opens a large space for investigating the fine structure of the spectra of single molecules and mixtures, representing a better way to assign frequencies to vibration modes through its neater and more precise numbers and to calculate the relationships between spectra. Infrared spectroscopy has been an important tool for studying polymorphism ${ }^{32}$ and is widely used by the pharmaceutical industry as a method to characterize polymorphs in APIs. ${ }^{32,33}$ As bioavailability has been shown to be dependent on the solid structure of the active molecule, the characterization of polymorphs in APIs remains an important issue in this context. The coincidence indexes calculated from the bar infrared spectra may contribute to this field through their capacity to translate spectra into numbers.

\section{Supplementary Information}

Supplementary information is available free of charge at http://jbcs.sbq.org.br as PDF file.

\section{Acknowledgments}

The authors thank the Laboratório de Análise, Departamento de Química Inorgânica, Instituto de Química, UFRJ. Our particular thanks go to Mrs Leonice B. Coelho and to Miss Glaucia W. Martins, for running the (many!) infrared spectra used in this paper. The authors also thank the Laboratório de Ressonância Magnética Nuclear, Departamento de Química Orgânica, for the carbon magnetic resonance spectra of the samples in the solid phase and in solution.

\section{References}

1. Wöhler, F.; Ann. Phys. Chem. 1829, 15, 619.

2. Wojtowicz, J. A.; Journal of the Swimming Pool and Spa Industry 2002, 4, 17. 
3. Budavari, S.; The Merck Index. An Encyclopedia of Chemicals, Drugs and Biologicals, $12^{\text {th }}$ ed.; Merck Research Laboratories: Rahway, 1966.

4. Tilstan, U.; Weinmann, H.; Org. Process Res. Dev. 2002, 6, 384.

5. Mendonça, G. F.; Magalhães, R. R.; de Mattos, M. C. S.; Esteves, P. M.; J. Braz. Chem. Soc. 2005, 16, 695.

6. Wojtowicz, J. A.; Encyclopedia of Chemical Technology, $4^{\text {th }}$ ed.; John Wiley \& Sons: New York, 1993.

7. Hill, H. W.; US pat. 3,898,222 A 1975.

8. Wojtowicz, J. A.; US pat. 4,498,921 and 4,472,187 1985.

9. Hiegel, G. A.; Peyton, K. B.; Synth. Commun. 1985, 15, 392.

10. de Luca, L.; Giacomelli, G.; Synlett 2004, 12, 2180.

11. Wengert, M.; Sanseverino, A. M.; de Mattos, M. C. S.; J. Braz. Chem. Soc. 2002, 13, 700.

12. Juenge, E. C.; Beal, D.; Duncan, W.; J. Org. Chem. 1970, 35 , 719.

13. Cunha, A. C.; Ferreira, V. F.; Quim. Nova 2006, 29, 520.

14. Mendonça, G. F.; Mattos, M. C. S.; Curr. Org. Synth. 2014, 10, 820 .

15. Newman, R.; Badger, R. M.; J. Am. Chem. Soc. 1952, 74, 3545.

16. Padgett, W. M.; Hamner, W. F.; available at http://www.kb.osu. edu/dspace/handle/1811/7531, accessed in January 2018.

17. Loughran, G.; Ehlers, G.; Crawford, W.; Burkett, J.; Ray, J.; Appl. Spectrosc. 1964, 83, 410.

18. Chen, W. C.; Wu, S. Y.; Liu, H. P.; Chang, C. H.; Chen, H. Y.; Chen, H. Y.; Tsai, C. H.; Chang, Y. C.; Tsai, F. J.; Man, K. M.; J. Clin. Lab. Anal. 2010, 24, 92.

19. Surinwong, S.; Prior, T. J.; Rujiwatra, A.; Chiang Mai J. Sci. 2014, 41, 414.

20. Barnett, S. A.; Blake, A. J.; Champness, N. R.; CrystEngComm 2003, 5, 134.

21. Yang, J.; Chen, Y.; Qin, S.; Liu, J.; Bi, C.; Liang, R.; Dong, T.; Feng, X.; Ind. Eng. Chem. Res. 2015, 54, 8048.
22. Tozetti, S. D. F.; de Almeida, L. S.; Esteves, P. M.; Mattos, M. C. S.; J. Braz. Chem. Soc. 2007, 18, 675.

23. Origin-Lab [CD-ROM], version 6.0; Microcal Software: Northampton, MA, USA, 1977.

24. Costa Neto, C.; Análise Orgânica. Métodos e Procedimentos para a Caracterização de Organoquímicos; Editora UFRJ: Rio de Janeiro, 2004.

25. Luo, Y.; Liu, Q.; Yang, L.; Wang, W.; Ling, Y.; Sun, B.; Res. Chem. Intermed. 2015, 41, 7059.

26. Tools for NMR Spectroscopists; available at http://www.nmrdb. org, accessed in January 2018.

27. Bernstein, J.; Polymorphism in Molecular Crystals; Clarendon Press: Oxford, 2002.

28. Andreeta, M. R. B. In Crystallization - Science and Technology; Andreeta, M. R. B., ed.; InTech: Croatia, 2012, p. 1-578.

29. Berthelot, M.; Repert. Chim. Applique 1859, 1, 284.

30. Patton, C. J.; Crouch, S. R.; Anal. Chem. 1977, 49, 464.

31. Spectral Database for Organic Compounds (SDBS); available at http://sdbs.db.aist.go.jp/sdbs/cgi-bin/cre_index.cgi, accessed in January 2018.

32. Brittain, H. G. In Polymorphism in Pharmaceutical Solids, $2^{\text {nd }}$ ed.; Brittain, H. G., ed.; Informa Healthcare: New York, 2009, p. 1-656.

33. Brittain, H. G.; J. Pharm. Sci. 1997, 86, 405.

Submitted: September 6, 2017 Published online: February 14, 2018 